\title{
BIORTHOGONALITY IN THE REAL SEQUENCE SPACES $\ell^{p}$
}

\author{
by ANTHONY J. FELTON and H. P. ROGOSINSKI* \\ (Received 14th August 1995)
}

\begin{abstract}
In this paper we generalise some of the results obtained in [1] for the $n$-dimensional real spaces $\ell^{p}(n)$ to the infinite dimensional real spaces $\ell^{p}$. Let $p>1$ with $p \neq 2$, and let $\mathrm{x}$ be a non-zero real sequence in $\ell^{p}$. Let $\mathcal{E}(\mathbf{x})$ denote the closed linear subspace spanned by the set $\{x\}^{ \pm}$of all those sequences in $\ell^{p}$ which are biorthogonal to $\mathrm{x}$ with respect to the unique semi-inner-product on $\ell^{p}$ consistent with the norm on $\ell^{p}$. In this paper we show that codim $\mathcal{E}(\mathbf{x})=1$ unless either $\mathbf{x}$ has exactly two non-zero coordinates which are equal in modulus, or $x$ has exactly three non-zero coordinates $\alpha, \beta, \gamma$ with $|\alpha| \geq|\beta| \geq|\gamma|$ and $|\alpha|^{p}>|\beta|^{p}+|\gamma|^{p}$. In these exceptional cases codim $\mathcal{E}(\mathbf{x})=2$. We show that $\{\mathbf{x}\}^{ \pm}$is a linear subspace if, and only if, $\mathbf{x}$ has either at most two non-zero coordinates or $x$ has exactly three non-zero coordinates which satisfy the inequalities stated above.
\end{abstract}

1991 Mathematics subject classification: 46C50.

\section{Introduction}

Throughout this paper, $p$ denotes a real number with $p>1$ and $p \neq 2$. Consider the real normed linear space $\ell^{p}$, and note that there exists a unique semi-inner-product on $\ell^{p}$ consistent with the norm. In fact for $\mathbf{x}, \mathbf{y} \in \ell^{p}$

$$
[\mathbf{x}, \mathbf{y}]=\frac{1}{\|\mathbf{y}\|^{p-2}} \sum_{i=1}^{\infty} x_{i}\left|y_{i}\right|^{p-1} \operatorname{sgn} y_{i}
$$

For a discussion of semi-inner-products and semi-inner-product spaces we refer the reader to [2] and [3]. The following definitions are given in [1]. If $x, y \in \ell^{p}$ then $x$ and $y$ are said to be biorthogonal if $[\mathbf{x}, \mathbf{y}]=[\mathbf{y}, \mathbf{x}]=0$. Further for fixed $\mathbf{x} \in \ell^{P}(n), \tau(\mathbf{x})$ is defined to be the number of elements in a maximal linearly independent set of vectors biorthogonal to $\mathbf{x}$. The following theorem is the main result (Theorem 4.5) of [1].

Theorem 0.1. Let $n \geq 2$, and let $\mathrm{x} \in \ell^{p}(n)$. Let $r$ be the number of non-zero coordinates of $\mathbf{x}$.

(i) If $r=0$ then $\tau(\mathbf{x})=n$.

(ii) If $r=1$ or $r \geq 4$ then $\tau(\mathbf{x})=n-1$.

- This research was funded by the University of Wales. 
(iii) If $r=2$ then $\tau(\mathbf{x})=n-1$ if the two non-zero coordinates have equal modulus, and $\tau(\mathbf{x})=n-2$ otherwise.

(iv) If $r=3$, let $\{\alpha, \beta, \gamma\}$ be a permutation of the three non-zero coordinates such that $|\alpha| \geq|\beta| \geq|\gamma|$. Then $\tau(\mathbf{x})=n-1$ if $|\alpha|^{p} \leq|\beta|^{p}+|\gamma|^{p}$ and $\tau(\mathbf{x})=n-2$ otherwise.

Definition 0.2. For $\mathbf{x} \in \ell^{p}$, define $\mathcal{E}(\mathbf{x})$ to be the smallest closed linear subspace in $\ell^{p}$ which contains every vector biorthogonal to $\mathbf{x}$.

Remark 0.3. Let $\mathbf{x} \in \ell^{p}$. Then $\mathcal{E}(\mathbf{x}) \subseteq\{\mathbf{y}:[\mathbf{y}, \mathbf{x}]=0\}$. (This follows immediately from the left-linearity and left-continuity of the semi-inner-product.)

In the next section we shall show that $\mathcal{E}(\mathbf{x})$ has finite codimension, and we shall determine codim $\mathcal{E}(\mathbf{x})$ for all non-zero $\mathbf{x}$ in $\ell^{p}$.

\section{The space $\mathcal{E}(\mathbf{x})$}

We introduce the following notation.

Notation. For $\mathbf{x}=\left(x_{1}, x_{2}, \ldots, x_{n}\right)$ in $\ell^{p}(n)$, denote by $\hat{\mathbf{x}}$ the sequence $\left(x_{1}, x_{2}, \ldots\right.$, $\left.x_{n}, 0,0, \ldots\right)$ in $\ell^{p}$. For $\mathbf{x}=\left(x_{1}, x_{2}, \ldots\right)$ in $\ell^{p}$, denote by $\mathbf{x}^{[n]}$ the sequence $\left(x_{1}, x_{2}, \ldots, x_{n}\right)$ in $\ell^{p}(n)$.

Theorem 1.1. Let $\mathbf{x}$ be a non-zero vector in $\ell^{p}$. Then $\operatorname{codim} \mathcal{E}(\mathbf{x})=1$ unless either

(i) $\mathbf{x}$ has exactly two non-zero coordinates $\alpha$ and $\beta$ with $|\alpha| \neq|\beta|$

or

(ii) $\mathbf{x}$ has exactly three non-zero coordinates $\alpha, \beta$ and $\gamma$ with $|\alpha| \geq|\beta| \geq|\gamma|$ and $|\alpha|^{p}>|\beta|^{p}+|\gamma|^{p}$.

If either of the conditions (i) or (ii) holds then $\operatorname{codim} \mathcal{E}(\mathbf{x})=2$.

Proof. Let $\mathbf{x} \in \ell^{p}$. Suppose first that $\mathbf{x}$ has infinitely many non-zero coordinates. Choose $N$ so that $\mathrm{x}^{[n]}$ has at least four non-zero coordinates when $n \geq N$. Then by Theorem 0.1 (ii) for $n \geq N, \tau\left(x^{[n]}\right)=n-1$, and we can find $(n-1)$ linearly independent vectors $\mathbf{f}_{i, n}(1 \leq i \leq n-1)$ in $\ell^{p}(n)$ which are biorthogonal to $\mathbf{x}^{[n]}$. The vector $\mathbf{x}^{[n]}$ is not a linear combination of these vectors since every such linear combination is leftorthogonal to $\mathbf{x}^{[n]}$. Hence $\left\{\mathbf{x}^{[n]}, \mathbf{f}_{1, n}, \ldots, \mathbf{f}_{n-1, n}\right\}$ is a basis for $\ell^{p}(n)$.

Let $\mathrm{y} \in \ell^{p}$, and let $n \geq N$. Then there exists scalars $\lambda_{i, n}(0 \leq i \leq n-1)$ so that

$$
\mathbf{y}^{[n]}=\lambda_{0, n} \mathbf{x}^{[n]}+\sum_{i=1}^{n-1} \lambda_{i, n} \mathbf{f}_{i, n} .
$$


By the left-linearity of the semi-inner-product,

$$
\left[\mathbf{y}^{[n]}, \mathbf{x}^{[n]}\right]=\lambda_{0, n}\left\|\mathbf{x}^{[n]}\right\|^{2}
$$

and so

$$
\lim _{n \rightarrow \infty} \lambda_{0, n}=\frac{[\mathbf{y}, \mathbf{x}]}{\|\mathbf{x}\|^{2}}
$$

Let

$$
\mathbf{z}_{n}=\sum_{i=1}^{n-1} \lambda_{i, n} \hat{\mathbf{f}}_{i, n}, \quad(n \geq N)
$$

Then $\mathbf{z}_{n} \in \mathcal{E}(\mathbf{x})$ since each of the vectors $\hat{\mathbf{f}}_{i, n, n}$ is biorthogonal to $\mathbf{x}$. By (1),

$$
\widehat{\mathbf{y}^{(n]}}=\lambda_{0, n} \widehat{\mathbf{x}^{(n]}}+\mathbf{z}_{n} \text {, }
$$

and so, using (3) and the observation that $\widehat{\mathbf{x}^{[n]}} \rightarrow \mathbf{x}$, and $\widehat{\mathbf{y}^{[n]}} \rightarrow \mathbf{y}$,

$$
\lim _{n \rightarrow \infty} \mathbf{z}_{n}=\mathbf{y}-\frac{[\mathbf{y}, \mathbf{x}]}{\|\mathbf{x}\|^{2}} \mathbf{x}
$$

Hence, since $\mathcal{E}(\mathbf{x})$ is closed,

$$
\mathbf{y}-\frac{[\mathbf{y}, \mathbf{x}]}{\|\mathbf{x}\|^{2}} \mathbf{x} \in \mathcal{E}(\mathbf{x})
$$

Noting that $\mathbf{x} \neq 0$, it follows from Remark 0.3 that $\mathbf{x} \notin \mathcal{E}(\mathbf{x})$. Since (5) holds for all $\mathbf{y}$ in $\ell^{p}$, we deduce that

$$
\ell^{p}=\mathcal{E}(\mathbf{x}) \oplus \mathcal{F}(\mathbf{x})
$$

where $\mathcal{F}(\mathbf{x})$ is the one-dimensional subspace generated by $\mathbf{x}$. It follows that $\operatorname{codim} \mathcal{E}(\mathbf{x})=1$.

Suppose now that $\mathbf{x}=\left(x_{1}, x_{2}, \ldots\right)$ has finitely many non-zero coordinates. Choose $n_{0}$ so that $x_{i}=0$ when $i>n_{0}$. Let $\tau_{0}=\tau\left(\mathbf{x}^{\left[n_{0}\right]}\right)$, and let $\tau_{1}=n_{0}-\tau_{0}$. Then we can find a basis $\left\{f_{1}, f_{2}, \ldots, f_{n_{0}}\right\}$ in $\ell^{p}\left(n_{0}\right)$ with $f_{i}$ biorthogonal to $x^{\left[n_{0}\right]}$ when $\tau_{1}+1 \leq i \leq n_{0}$. Let $\mathbf{y}=\left(y_{1}, y_{2}, \ldots\right) \in \ell^{p}$. Then there exist scalars $\lambda_{i}\left(1 \leq i \leq n_{0}\right)$ so that

$$
\mathbf{y}=\sum_{i=1}^{n_{0}} \lambda_{i} \hat{\mathbf{f}}_{i}+\sum_{i=n_{0}+1}^{\infty} y_{i} \mathbf{e}_{i},
$$


where $\mathbf{e}_{i}$ is the $\mathrm{i}^{\text {th }}$ standard basis vector in $\ell^{p}$. We can write

$$
\mathbf{y}=\mathbf{y}_{1}+\mathbf{y}_{2}
$$

where

$$
\mathbf{y}_{1}=\sum_{i=\tau_{1}+1}^{n_{0}} \lambda_{i} \hat{\mathbf{f}}_{i}+\sum_{i=n_{0}+1}^{\infty} y_{i} \mathbf{e}_{i}, \quad \text { and } \quad \mathbf{y}_{2}=\sum_{i=1}^{\tau_{1}} \lambda_{i} \hat{\mathbf{f}}_{i} .
$$

Since each of the vectors $\hat{\mathbf{f}}_{i}\left(\tau_{1}+1 \leq i \leq n_{0}\right)$ and each of the vectors $\mathbf{e}_{i}\left(i \geq n_{0}+1\right)$ is biorthogonal to $\mathbf{x}$,

$$
\mathbf{y}_{1} \in \mathcal{E}(\mathbf{x})
$$

If $\mathcal{F}(\mathbf{x})$ is the $\tau_{1}$-dimensional subspace of $\ell^{p}$ spanned by the vectors $\hat{\mathbf{f}}_{i}\left(1 \leq i \leq \tau_{1}\right)$ then

$$
\mathbf{y}_{2} \in \mathcal{F}(\mathbf{x})
$$

Noting that the map $z \rightarrow z^{\left.\eta_{0}\right]}$ is a continuous linear map from $\stackrel{\rho p}{.}$ onto $\ell^{p}\left(n_{0}\right)$, and also that $\mathbf{z}$ is biorthogonal to $\mathbf{x}$ if, and only if, $\mathbf{z}^{\left[n_{0}\right]}$, is biorthogonal to $\mathbf{x}^{\left[n_{0}\right]}$, it is easy to see that if $\mathbf{z} \in \mathcal{E}(\mathbf{x})$ then $\mathbf{z}^{\left[n_{0}\right]} \in \mathcal{E}\left(\mathbf{x}^{\left[n_{0}\right]}\right)$. Now let $\mathbf{z} \in \mathcal{E}(\mathbf{x}) \cap \mathcal{F}(\mathbf{x})$. Then $\mathbf{z}^{\left[n_{0}\right]}$ belongs to $\mathcal{E}\left(\mathbf{x}^{\left[n_{0}\right]}\right)$, and so $\mathrm{z}^{\left[n_{0}\right]}$ is a linear combination of the vectors $\mathbf{f}_{i}\left(\tau_{1}+1 \leq i \leq n_{0}\right)$. On the other hand, since $\mathrm{z}$ is a linear combination of the vectors $\hat{\mathbf{f}}_{i}\left(1 \leq i \leq \tau_{1}\right), \mathrm{z}^{\mathbf{n}_{0} \mathrm{l}}$ must also be a linear combination of the vectors $\mathrm{f}_{i}\left(1 \leq i \leq \tau_{1}\right)$. It follows that $\mathrm{z}^{\left[n_{0}\right)}$ is the zero-vector in $\ell^{p}\left(n_{0}\right)$, and so $\mathbf{z}\left(=\overline{\left.\mathbf{z}^{\left(m_{0} \mid\right.}\right)}\right)$ is the zero-vector in $\ell^{p}$. Hence

$$
\mathcal{E}(\mathbf{x}) \cap \mathcal{F}(\mathbf{x})=\{\mathbf{0}\} .
$$

By (7), (8), (9) and (10) we see that

$$
\ell^{p}=\mathcal{E}(\mathbf{x}) \oplus \mathcal{F}(\mathbf{x}),
$$

and hence codim $\mathcal{E}(\mathbf{x})=\operatorname{dim} \mathcal{F}(\mathbf{x})=\tau_{1}$. An application of Theorem 0.1 shows that $\tau_{1}=1$ unless $\mathbf{x}$ satisfies either of the conditions (i) and (ii) in which case $\tau_{1}=2$.

Remark 1.2. If $E$ and $F$ are proper linear subspaces of $X$ with codim $E=1$ and $E \subseteq F$ then $E=F$.

Let $[.$, . ] be a semi-inner-product on the normed space $X$ which is consistent with the norm on $X$.

Theorem 1.3. (i) Let $\mathbf{x}$ be a non-zero vector in $X$. Then $\mathcal{E}(\mathbf{x})$ has codimension 1 in $X$ if, and only if, 


$$
\mathcal{E}(\mathbf{x})=\{\mathbf{y} \in X:[\mathbf{y}, \mathbf{x}]=0\} .
$$

(ii) If the set of all vectors biorthogonal to $\mathbf{x}$ is a linear subspace of $X$ with codimension 1 then every vector which is left-orthogonal to $\mathbf{x}$ is also right-orthogonal to $\mathbf{x}$.

Proof. Write $\{\mathbf{x}\}^{L}=\{\mathbf{y} \in X:[\mathbf{y}, \mathbf{x}]=0\}$.

(i) If $\mathcal{E}(\mathbf{x})=\{\mathbf{x}\}^{L}$ then $\mathcal{E}(\mathbf{x})$ is the kernel of a non-zero continuous linear functional on $X$, and so has codimension 1 . Suppose conversely that $\mathcal{E}(\mathbf{x})$ has codimension 1 . Let $E=\mathcal{E}(\mathbf{x})$ and $F=\{\mathbf{x}\}^{L}$. Applying Remarks 0.3 and 1.2 we see that $\mathcal{E}(\mathbf{x})=\{\mathbf{x}\}^{L}$.

(ii) Apply Remark 1.2 with $E=\{\mathbf{y}: \mathbf{x} \pm \mathbf{y}\}$ and $F=\{\mathbf{x}\}^{L}$.

\section{The subspace problem}

For $\mathbf{x} \in \ell^{p}$, let $\{\mathbf{x}\}^{ \pm}$denote the set of all those sequences in $\ell^{p}$ which are biorthogonal to $x$. In this section we consider the problem of characterising those $x$ for which $\{x\}^{ \pm}$ is a linear subspace. We begin with the following lemma.

Lemma 2.1. (i) Let $\mathrm{x} \in \ell^{p}(3)$. If all of the coordinates of $\mathrm{x}$ are non-zero then there exists a vector in $\ell^{p}(3)$ which is left-orthogonal but not right-orthogonal to $\mathbf{x}$.

(ii) Let $\mathbf{x} \in \ell^{p}$. If $\mathbf{x}$ has at least three non-zero coordinates then there exists a vector in $\ell^{p}$ which is left-orthogonal but not right-orthogonal to $\mathbf{x}$.

Proof. We shall only prove (i) since (ii) then follows as an obvious consequence.

Noting the fact that the semi-inner-product is homogeneous, we can assume without loss of generality that $\mathbf{x}=(a, b, 1)$, with $a$ and $b$ non-zero. Suppose for a contradiction that every vector which is left-orthogonal to $\mathbf{x}$ is right-orthogonal to $\mathbf{x}$. The vector $\left(1,0,-|a|^{p-1} \operatorname{sgn} a\right)$ is left-orthogonal to $\mathbf{x}$, and so by our supposition right-orthogonal to $x$. This implies that

$$
a+|a|^{(p-1)^{2}} \operatorname{sgn}(-\operatorname{sgn} a)=0,
$$

and so $|a|=|a|^{(p-1)^{2}}$. Hence $|a|=1$ since $p \neq 2$. Similarly the left-orthogonality and consequent right-orthogonality of $\left(0,1,-|b|^{p-1} \operatorname{sgn} b\right)$ to $\mathbf{x}$ implies that $|b|=1$. Since $|a|=|b|=1$, the vector $(2 \operatorname{sgn} a,-\operatorname{sgn} b,-1)$ is left-orthogonal to $\mathbf{x}$, and a simple calculation shows that the right-orthogonality of this vector to $\mathbf{x}$ implies that $2^{p-1}=2$. Since $p \neq 2$ we obtain the desired contradiction.

Theorem 2.2. For given $\mathbf{x} \in \ell^{p},\{\mathbf{x}\}^{ \pm}$is a linear subspace if, and only if, either $\mathbf{x}$ has at most two non-zero coordinates or $\mathrm{x}$ has exactly three non-zero coordinates $\alpha, \beta, \gamma$ with $|\alpha| \geq|\beta| \geq|\gamma|$ and $|\alpha|^{p}>|\beta|^{p}+|\gamma|^{p}$.

Proof. If $\mathbf{x}=0$ then $\{\mathbf{x}\}^{ \pm}=\ell^{p}$. If $\mathbf{x}$ has exactly one non-zero coordinate $x_{n_{1}}$ then 


$$
\{\mathbf{x}\}^{ \pm}=\left\{\left(y_{1}, y_{2}, \ldots\right) \in \ell^{p}: y_{n_{1}}=0\right\} .
$$

If $\mathbf{x}$ has exactly two non-zero coordinates $x_{n_{1}}$ and $x_{n_{2}}$ then it is easily verified that

$$
\{\mathbf{x}\}^{ \pm}=\left\{\left(y_{1}, y_{2}, \ldots\right) \in \ell^{p}: y_{n_{1}}=y_{n_{2}}=0\right\} \text { if }\left|x_{n_{1}}\right| \neq\left|x_{n_{2}}\right|,
$$

and

$$
\{\mathbf{x}\}^{ \pm}=\left\{\left(y_{1}, y_{2}, \ldots\right) \in \ell^{p}: y_{n_{1}}=-\operatorname{sgn}\left(\frac{x_{n_{1}}}{x_{n_{2}}}\right) y_{n_{2}}\right\} \quad \text { if } \quad\left|x_{n_{1}}\right|=\left|x_{n_{2}}\right| .
$$

Hence $\{\mathbf{x}\}^{ \pm}$is a linear subspace if $\mathbf{x}$ has at most two non-zero coordinates. If $\mathbf{x}$ has exactly three non-zero coordinates $x_{n_{1}}, x_{n_{2}}$ and $x_{n_{3}}$ with $\left|x_{n_{1}}\right| \geq\left|x_{n_{2}}\right| \geq\left|x_{n_{3}}\right|$ and $\left|x_{n_{1}}\right|^{p}>\left|x_{n_{2}}\right|^{p}+\left|x_{n_{3}}\right|^{p}$ then $\tau\left(x_{n_{1}}, x_{n_{2}}, x_{n_{3}}\right)=1$ and

$$
\{\mathbf{x}\}^{ \pm}=\left\{\left(y_{1}, y_{2}, \ldots\right) \in \ell^{p}:\left(y_{n_{1}}, y_{n_{2}}, y_{n_{3}}\right) \in \mathcal{V}\right\},
$$

where $\mathcal{V}$ is the one-dimensional linear subspace of $\ell^{p}(3)$ consisting of all those vectors which are biorthogonal to $\left(x_{n_{1}}, x_{n_{2}}, x_{n_{3}}\right)$. Hence also in this case $\{\mathbf{x}\}^{ \pm}$is a linear subspace.

In all of the remaining cases, Lemma 2.1 shows that there exists a vector which is left-orthogonal but not right-orthogonal to $\mathbf{x}$. Moreover in all of these cases Theorem 1.1 shows that codim $\mathcal{E}(\mathbf{x})=1$. Hence $\{\mathbf{x}\}^{ \pm}$is not a linear subspace, since otherwise $\mathcal{E}(\mathbf{x})=\{\mathbf{x}\}^{ \pm}$and Theorem 1.3(ii) leads to a contradiction.

Acknowledgement. We would like to thank the referee for his helpful comments concerning the presentation of this paper.

\section{REFERENCES}

1. Anthony J. Fexton and H. P. Rogosinski, Biorthogonality in the Banach Spaces $\ell^{p}(n)$, Proc. Edinburgh Math. Soc. 39 (1996), 325-336.

2. J. R. Giles, Classes of semi-inner-product spaces, Trans. Amer. Math. Soc. 129 (1967), 436-446.

3. G. Lumer, Semi-inner-product spaces, Trans. Amer. Math. Soc. 100 (1961), 29-43.

Department of Mathematics

UNIVERSITY OF WaLES, SWANSEA

SINGLETON PARK, SA2 8PP

WALES 